\title{
Cognitive Inhibitory Control Capacities Revealed by Cross-Language Priming
}

\author{
Ewald Neumann* \\ University of Canterbury, New Zealand
}

*Corresponding author: Ewald Neumann, University of Canterbury, Christchurch, New Zealand.

Received Date: November 01, 2018

Published Date: December 03, 2018

\begin{abstract}
Language access in bilinguals requires highly refined selective attention abilities. This review chronicles the implications from cross-language priming studies collected from three different continents. These research explorations using novel selective attention paradigms begin to reveal the local word and global language inhibitory control capacities the bilingual brain uses to modulate its languages. Neurological and clinical implications regarding inhibitory mechanisms and the potential applicability to mental control deficits are also raised.
\end{abstract}

\section{Mini Review}

Selective attention processes are necessary for navigating through the constant stream of visual information competing for our attention on a moment-to-moment basis but are especially critical in rapidly changing situations where briefly designated targets of attention are in conflict with rival non-target distractors. How our attentional mechanisms overcome the competition of conflicting stimuli and selectively focus on target information has become an increasingly important research topic across several intersecting domains. Exploring how cross-language inhibitory processes work in the bilingual brain provides unique insight into some of the parameters and consequences involved with inhibitory control. The implications from cross-language studies that track priming costs and benefits of translation equivalent words that were either used as target words or non-target distractor words are chronicled below, but first a general description of the paradigm used follows.

The efficiency of processing simultaneously presented target and distractor stimuli in a selective attention task can be positively (positive priming) or negatively (negative priming) influenced by the relationship these target and distractor stimuli have with stimuli in the previous display. In a traditional negative priming task, participants see two sequentially presented static displays: a prime display followed by a probe display, each consisting of a target and a distractor. A negative priming effect or detriment occurs when responses to the target in a probe display are slower or more error-prone when a previously ignored prime distractor becomes a subsequent probe target than in the neutral condition with no relationship between prime and probe targets and distractors. The negative priming effect indicates that a successful prime selection involves the processing of the distractor to the extent that it can produce a reaction time cost upon subsequent presentation as a target [1,2]. Different stimuli have been used in negative priming tasks, as well as different manipulations involving the conceptual relationship between the non-target distractors and their subsequent presentation as a target [3]. These may vary from identity (e.g., ignoring a prime distractor letter "Z" that becomes the subsequent probe target letter "Z") to various forms of semantic relationships, such as ignoring the picture of a hammer and responding subsequently to the semantically related word "screwdriver" [2,4], or ignoring the word "APPLE" and responding next to the word "manzana", which is the Spanish translation of the English word "apple" for English-Spanish bilinguals in a crosslanguage priming task [5].

The experiments that provide the main source for the present mini review investigated negative priming using a new paradigm [6]. Instead of concurrently presented target and non-target stimuli in the prime display, followed by concurrently presented target and non-target stimuli in the probe display, we combined the traditional negative priming paradigm with rapid serial visual presentation (RSVP) typically used in studies that explore the temporal limitation of attentional selection [7]. In the new RSVPNP paradigm, which is designed to probe a variety of semantic and 
cross-language negative priming effects, the prime and probe trial each consists of a stream of stimuli presented sequentially in rapid succession at the same location. Because the prime distractor and the probe target appear at the same spatial location, this paradigm allows researchers to study how target selection is accomplished when the target and distractor overlap spatially but are separated temporally.

In the four experiments using the RSVP-NP task to investigate negative priming we included a mixture of stimulus types (letters, digits, English number words, and logographic Chinese number words) which are presented in two rapid serial visual presentation (RSVP) streams with prime and probe trials consisting of a stream of stimuli presented sequentially in rapid succession at the same location with the relationship between the distractor in the prime trial and the target in the probe trial systematically altered to investigate how target selection is accomplished when the target and distractor overlap spatially, but are separated temporally (Figure 1 shows an example of stimuli displays from one of the experiments, and Figure 2 shows the results).

In Experiments 1 and 2, the target and distractor within each trial were either two digits or two number words; whereas in Experiment 3 two different representational forms were used (digits and logographic Chinese number words). In Experiment 4 a cross-language manipulation with Chinese-English bilinguals was used and prime distractors and probe targets switched from a subject's dominant (Chinese) language to their non-dominant (English) language to investigate bilingual visual-linguistic control mechanisms (Figure 1 \& Figure 2).

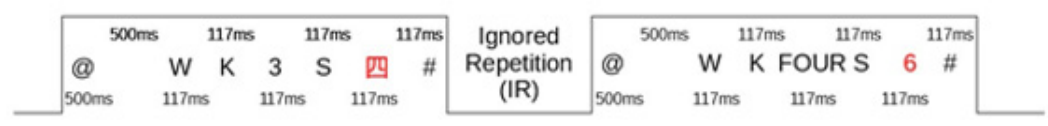

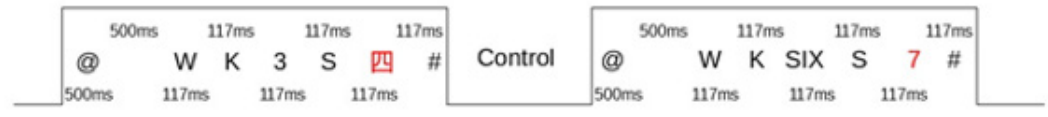

TIME

Figure 1: This figure shows a sample sequence of items in a prime stream (on left) and a probe stream (on right) for an ignored Repetition (IR) condition (top) and control condition (bottom). Participants are always required to respond to the target black numerical and ignore the non-target, conflicting red numerical. The numerical can be verbal in the form of a word, or in the form of a number. For the IR condition in this example, note that the red distractor in the prime stream is the Chinese word for Four. If the probe response to the English word Four in the probe stream is delayed, compared to the control condition, that cost would indicate negative priming between different languages. The timing of each event in streams is also shown in milliseconds (ms). Each item appears sequentially in the middle of a computer screen.

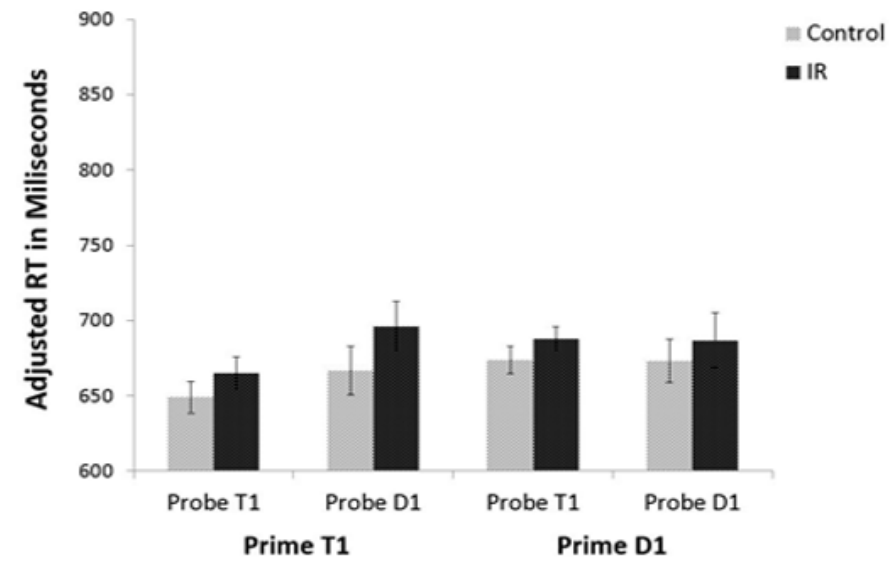

Figure 2: T1 refers to the target item's position in the prime or probe stream, whereas D1 refers to the distractor item's position in the prime or probe stream. As can be seen, the black bars show a consistent response time delay in the ignored repetition (IR), condition, compared to the control condition. This clearly indicates that regardless if the non-target distractor item appears before or after the target item in the prime stream or probe stream, there is always a reaction time cost. This delay represents the negative priming effect.

The results from all of these experiments show the robustness of negative priming under RSVP by extending the finding of negative priming using different semantically related stimuli in the prime and probe streams. It is noteworthy that the ignored non-target competing item in the prime stream is only associatively related by meaning when it becomes the sought-after target in the probe 
stream. This makes the impairment in responding to it particularly important for understanding the mechanisms the brain uses to process distracting information.

The main discovery here is that there is an opposing flipside to accessing wanted information. The mechanism revealed by our research inhibits unwanted information to the extent that the suppression leads to closely associated concepts in one's mind becoming less accessible than a similar item would be which had not recently been encountered in the prime stream. This even happens between translations of concepts across different languages $[5,8]$. These remarkable findings are also in total agreement with brain cell recording research that has begun to address the neural substrates underlying target and distractor processing [9]. This brain cell research suggests that participants control neurons representing targeted items independently of those representing non-target objects by enhancing the firing of neurons that have a preference for the target, while actively inhibiting or suppressing those that encode the non-target. The lingering effects of the neuronal inhibition of distracting information, which was employed to efficiently focus on essential relevant information, can be seen in our often perplexingly slower processing and responses when irrelevant distracting information suddenly becomes relevant.

Cross-language experiments that have used more traditional static prime and probe displays each containing a target and distractor, instead of RSVP streams $[5,8]$ provide additional supportive evidence of ignored repetition negative priming between translation equivalents across languages. They also show the extensive reach of inhibitory control by the surprising concurrent finding of no evidence of attended repetition positive priming of translation equivalents. The lack of a priming benefit in this context indicates that the potential spreading activation "positive" priming of a probe target word from a translation equivalent prime target word was completely eliminated, because of the global inhibition of the now conflicting non-target language. The remarkably consistent findings in these studies with different groups of bilinguals strongly imply that two forms of inhibition were in operation: one at the local prime distractor word level and the other at the global prime language level. Thus, local word-based inhibition led to crosslanguage ignored repetition negative priming when the previous distractor was the translation equivalent of the subsequent probe target, and global-level inhibition led to the elimination of cross-language attended repetition positive priming, because the prime language became irrelevant and potentially distracting for responding in the language required for the probe target word. This work, especially in combination with the cross-language findings observed by Li et al. [6], suggests that active suppression of irrelevant distracting information is a more ubiquitous form of cognitive control than previously thought. In fact, it even appears to be fully intact in young children [10-12] and elderly adults [13].

There are several additional potentially important theoretical and empirical implications highlighted by our cross-language studies with bilinguals. Earlier studies have attempted to draw parallels between selective attention and memory research via an inhibitory or suppressive information processing mechanism attention and memory may share in common [14-16]. As described previously, this inhibitory mechanism is thought to suppress distracting, non-target words in negative priming tasks. A similarly described active suppressive mechanism has also been posited to accommodate two different memory phenomena: retrieval induced forgetting $[14,17]$ and the "no-think" component of the Think/Nothink $(\mathrm{T} / \mathrm{NT})$ task $[18,19]$. Establishing that a similar or perhaps the same active inhibitory mechanism is involved in reducing or eliminating interference effects from no longer relevant words in each of these cases would help advance and unite both the selective attention and memory literatures through a shared processing mechanism.

From our perspective, another way to accommodate the elimination of positive priming across languages in a task that nevertheless producesnegative priming acrosslanguages is to realize that it is a response time analogue to the "no-think" component of the T/NT phenomenon in the memory literature [18-21]. Notably, the T/NT task involves a reminder of an unwanted memory of a previously encountered word and instructions to suppress the thought of that word from awareness without mentioning the word itself. Similarly, with regard to the cross-language priming tasks by Neumann et al. [5] and Nkrumah \& Neumann [8], participants are simultaneously induced not to think of a language that is attended in the prime display nor the non-target distracting word when both become irrelevant and potentially distracting prior to the onset of the probe display. Rather than being instructed not to think about a word, as in the T/NT task, people are being induced not to think about a language on the one hand and a conflicting word on the other by the regular alternation between languages and the nontarget status of the ignored prime word prior to the impending response to the probe target word.

These cross-language experiments [5,8] provide evidence of suppressive processing at both the local and global level that is potentially detectable on an almost trial by trial basis, especially for those bilinguals who are more proficient in their non-dominant language [5]. Such inhibitory mechanisms help to illuminate how selective attention harnesses the words and languages of proficient bilinguals. The observed priming effects should therefore become particularly valuable tools for providing alternative ways of evaluating the neurobiological role of GABAergic metabolism whenever inhibitory information processing is being exploited in order to efficiently suppress unwanted memories whether they stem exogenously from the environment in a selective attention task or endogenously in a memory task. Schmitz and colleagues, for example, observed that hippocampal GABA (a chemical neurotransmitter substance that implements neural inhibition) contributes to stopping unwanted memories [21]. They showed that GABAergic inhibition of hippocampal retrieval activity forms the key link in the volitional inhibitory control underlying thought suppression and, crucially, the memory for suppressed content. Their evidence for a mechanism enabling inhibitory control over specific memories via GABAergic inhibition of local hippocampal activity could provide an underpinning mechanism for the total absence of attended repetition positive priming combined with robust ignored repetition negative priming in our crosslanguage paradigms [5,8]. Establishing a firmer linkage among these purportedly suppressive selective attention and memory phenomena within a neurobiological framework should be intensively pursued. 
Other bilingual researchers have begun accepting that local word and global language suppressive processing mechanisms can simultaneously modulate languages and the words within them in bilinguals [22-24]. As far as we are aware, however, the cross-language experiments reviewed here provide the only empirical evidence in the domain of bilingualism that reveal both such inhibitory processes in the same cross-language task. The novel nature of our cross-language selective attention paradigms may be necessary to reveal the dynamics of bilingual language regulation in ways that may not be readily available with the singularly presented stimulus items, used in the vast majority of bilingual research. Perhaps nowhere is this better exemplified than by having bilinguals dichotomized into the less and more proficient in their second language $[5,25]$. Differences between these more and less proficient bilinguals reinforce and extend our conjectures by showing that both the elimination of cross-language attended repetition positive priming coupled with greatly heightened ignored repetition negative priming are especially prominent in the more proficient bilinguals, compared to the less proficient. From our perspective, the best explanation for these findings is that they demonstrate a finely-tuned interplay between independent excitatory and inhibitory mechanisms. These mechanisms each have their own capacity limitations, which manifest in distinctly different patterns of priming for the less and more proficient. These capacity limitations are impacted by the proficiency level of the bilingual in much the same way expertise in any domain streamlines thought processes [26]. The different priming patterns reveal the consequences of implementing different degrees of excitation and suppression as a function of second language proficiency, ultimately manifesting for instance in the virtuosity with which proficient bilinguals can vacillate so effortlessly between their languages.

One of the main goals of cognitive science according to Pylyshyn [27] is to establish genuine information processing mechanisms that are not too remote from actual neurophysiological mechanisms of the brain. Mutual verifications from studies showing suppressive priming effects with words and no-think memory effects with words can help establish such a psychologically real information processing mechanism. This selective suppressive attentional mechanism gives rise to one side of the finely-tuned interplay between independent excitatory and inhibitory mechanisms with the particularly vital role of momentarily purging conflicting unwanted memories or thoughts $[15,16,20]$.

As mentioned earlier, attentional inhibition has also been more directly probed through brain cell recordings taken while subjects engage in a visual selective attention task. This research provides further insight into how unwanted information is inhibited and target information enhanced by revealing the neural substrates involved in using inhibition and activation to mentally disentangle concurrently presented targets from non-target stimuli [9]. Their findings clearly imply that the brain controls neuronal activation and suppression by independently enhancing the firing of targetpreferenced neurons and inhibiting or actively suppressing neurons that encode non-targets. Cerf and colleagues' experiments can be seen as a milestone in selective attention research, because they help corroborate the findings of many decades of research and theoretical work by cognitive scientists positing equally vital roles for target activation and non-target inhibition in selective attention processing $[15,16,28]$.

The Cerf et al. [9] neurophysiological study clearly substantiates the involvement of active suppression of the distractor representation in resolving target and distractor conflict in selective attention tasks. It showed that dissociated neural responses of neural ensembles encoding concurrently overlapping target and distractor stimuli were characterized by distinctly different neural dynamics. More specifically, neurons that had a preference for a current target object showed heightened activity, whereas neurons that had a preference for the current non-target distractor stimulus were actively suppressed. This suppressive processing happened in a particularly informative and original manner. There was no mere reduction in neural firing rate for the neurons sensitive to the conflicting distractor object, nor did the firing rate merely reduce to spontaneous baseline rates of firing when the preferred stimulus was not present. Rather, while the competition between the current target and non-target distractor stimulus was being resolved, the firing rate of the neurons with a preference for the distractor reduced their firing rate to below their spontaneous baseline rate. This potentially provides a first order or proximal causal mechanism underpinning conflict resolution by selective distractor inhibition. As such, the active suppression of conflicting distractor representations may underpin the root cause of the consequences of such inhibition in the negative priming phenomena elucidated in our within and cross-language experiments [6,29].

Taken collectively, the discoveries reviewed here should eventually help to provide not only more nuanced approaches for explaining bilingual language modulation, but also mental problems that appear to involve underlying cognitive inhibitory control issues such as obsessive-compulsive disorder (OCD). More accurate explanations of the underpinning causal mechanisms of OCD, posttraumatic stress disorder [30], and other more devastating mental control problems involving impaired inhibitory processing, such as schizophrenia [31,32], should ultimately lead to better treatments and successful treatment outcomes.

\section{Conclusion}

Our bilingual research reveals the flexibility and robustness of inhibitory mechanisms working together with excitatory mechanisms to enable us to manoeuvre through distracting information and focus on what is momentarily relevant. A bilingual's ability to inhibit either whole languages or a particularly conflicting word when they are distracting in a current situation provides a unique insight into the brain's selective processing capacities. The confirmation of the mechanisms uncovered by our findings are supported by research involving brain cell recording and by neural inhibition explorations of hippocampal GABA's contribution to the volitional avoidance of unwanted memories.

\section{Acknowledgement}

None.

\section{Conflict of Interest}

No conflict of interest. 


\section{References}

1. Neumann E, Levin JR (2018) Can the use of seven key manipulations and predicted pattern testing bring more clarity to negative priming investigations? American Journal of Psychology 131(1): 3-18.

2. Tipper SP, Driver J (1988) Negative priming between pictures and words in a selective attention task: Evidence for semantic processing of ignored stimuli. Mem Cognit 16(1): 64-70.

3. Frings C, Schneider KK, Fox E (2015) The negative priming paradigm: An update and implications for selective attention. Psychon Bull Rev 22(6): 1577-1597.

4. Tipper SP (1985) The negative priming effect: Inhibitory priming by ignored objects. Q J Exp Psychol A 37(4): 571-590.

5. Neumann E, McCloskey MS, Felio AC (1999) Cross-language positive priming disappears, negative priming does not: Evidence for two sources of selective inhibition. Memory \& Cognition 27(6): 1051-1063.

6. Li L, Neumann E, Chen Z (2017) Identity and semantic negative priming in rapid serial visual presentation streams. Atten Percept Psychophys 79(6): 1755-1776.

7. Dux PE, Marois R (2009) How humans search for targets through time A review of data and theory from the attentional blink. Atten Percept Psychophys 71(8): 1683-1700.

8. Nkrumah IK, Neumann E (2018) Cross-language negative priming remains intact, while positive priming disappears: Evidence for two sources of selective inhibition. Journal of Cognitive Psychology 30: 361384.

9. Cerf M, Thiruvengadam N, Mormann F, Kraskov A, Quiroga RQ, et al (2010) On-line, voluntary control of human temporal lobe neurons Nature 467(7319): 1104-1108.

10. Pritchard VE, Neumann E (2004) Negative priming effects in children engaged in nonspatial tasks: Evidence for early development of an intact inhibitory mechanism. Dev Psychol 40: 191-203.

11. Pritchard VE, Neumann E (2009) Avoiding the potential pitfalls of using negative priming tasks in developmental studies: Assessing inhibitory control in children, adolescents and adults. Dev Psychol 45(1): 272-283.

12. Pritchard VE, Neumann E (2011) Classic Stroop negative priming effects for children and adults diverge with less-conflicting and nonconflicting conditions. Am J Psychol 124(4): 405-419.

13. Schooler C, Neumann E, Caplan LJ, Roberts BR (1997a) Continued inhibitory capacity throughout adulthood: Conceptual negative priming in younger and older adults. Psychol Aging 12(4): 667-674.

14. Anderson MC, Spellman BA (1995) On the status of inhibitory mechanisms in cognition: memory retrieval as a model case. Psychol Rev 102(1): 68-100.

15. Neumann E, Cherau JF, Hood KL, Steinnagel SL (1993) Does inhibition spread in a manner analogous to spreading activation? Memory 1(2): 81-105.

16. Neumann E, DeSchepper BG (1992) An inhibition-based fan effect: Evidence for an active suppression mechanism in selective attention. Canadian Journal of Psychology 46(1): 1-40.
17. Buckley ME, Neumann E (2018) Retrieval induced forgetting and enhancement in tertiary law examinations: Are Law Students Unique? Psychology \& Psychological Research International Journal 3(1): 1-15.

18. Anderson MC, Green C (2001) Suppressing unwanted memories by executive control. Nature 410(6826): 366-369.

19. Anderson MC, Ochsner KN, Kuhl B, Cooper J, Robertson E, et al. (2004) Neural Systems Underlying the Suppression of Unwanted Memories. Science 303(5655): 232-235.

20. Neumann E, Nkrumah IK, Chen Z (2018a) Excitatory and inhibitory priming by attended and ignored non-recycled words with monolinguals and bilinguals. Memory 26(9): 1244-1255.

21. Schmitz TW, Correia MM, Ferreira CS, Prescot AP, Anderson MC (2017) Hippocampal GABA enables inhibitory control over unwanted thoughts. Nat Commun 8(1): 1311

22. Guo T, Liu H, Misra M, Kroll JF (2011) Local and global inhibition in bilingual word production: FMRI evidence from Chinese-English bilinguals. Neuro Image 56(4): 2300-2309.

23. Misra M, Guo T, Bobb SC, Kroll JF (2012) When bilinguals choose a single word to speak: Electrophysiological evidence for inhibition of the native language. J Mem Lang 67: 224-237.

24. Misra M, Guo T, Bobb SC, Kroll JF (2012) When bilinguals choose a single word to speak: Electrophysiological evidence for inhibition of the native language. J Mem Lang 67: 224-237.

25. Neumann E, Nkrumah IK, Chen Z (2018b) Second language proficiency effects on cross-language positive and negative priming in Twi-English bilinguals. Journal of Clinical Psychology and Cognitive Science 2(1): 8-16.

26. Lewis RL, Vasishth S (2005) An Activation-Based Model of Sentence Processing as Skilled Memory Retrieval. Cogn Sci 29(3): 375-419.

27. Pylyshyn ZW (1984) Computation and cognition: toward a foundation for cognitive science. Cambridge, MA: MIT Press.

28. Neumann E, De Schepper BG (1991) Costs and benefits of target activation and distractor inhibition in selective attention. J Exp Psychol Learn Mem Cogn 17(6): 1136-1145.

29. Neumann E (2018) Bilingual cross-language priming reveals common inhibitory modulation effects in selective attention and memory. Journal of Brain Behavior and Cognitive Sciences 1(2): 9.

30. Catarino A, Küpper CS, Werner-Seidler A, Dalgleish T, Anderson MC (2015) Failing to Forget : Inhibitory-control deficits compromise memory suppression in posttraumatic stress disorder. Psychological Science 26(5): 604-616.

31. Schooler C, Neumann E, Caplan LJ, Roberts BR (1997b) A time course analysis of Stroop interference and facilitation: Comparing normal individuals and individuals with schizophrenia. J Exp Psychol Gen 126: 19-36.

32. Schooler C, Neumann E, Caplan LJ, Roberts BR (1997c) Stroop theory, memory, prefrontal cortical functioning: Reply to Cohen et al. (1997). J Exp Psychol Gen 126(1): 42-44. 